\title{
Las competencias digitales de los futuros docentes. Un análisis con estudiantes de Magisterio de Educación Infantil de la Universidad de Alicante ${ }^{1}$
}

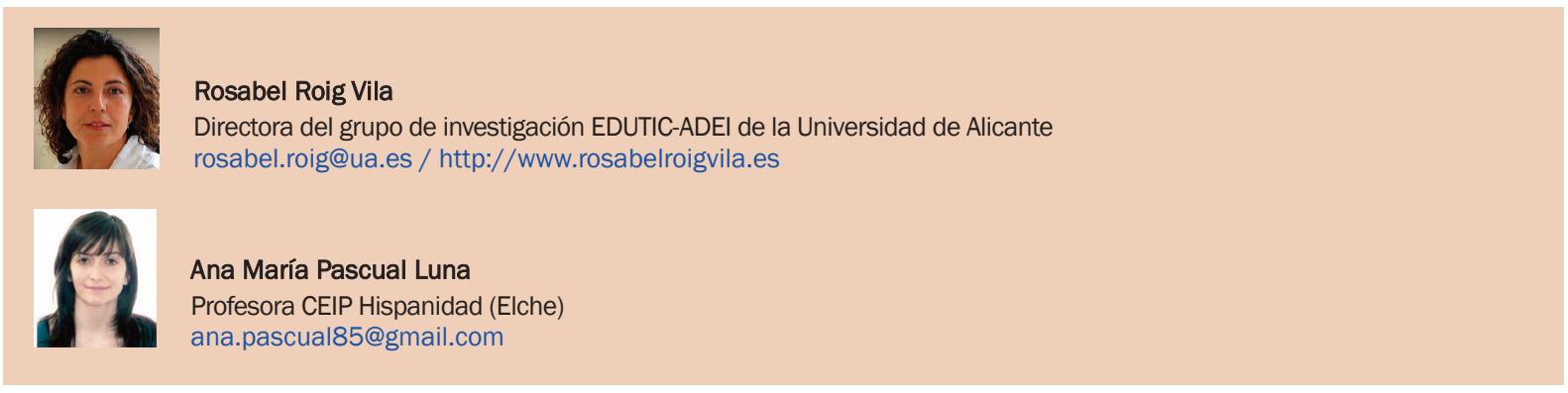

|Fecha presentación: 10/10/2012 | Aceptación: 22/11/2012 |Publicación: 20/12/2012

\begin{abstract}
Resumen
En el presente trabajo se detalla el estudio descriptivo realizado sobre las competencias digitales en alumnos de Magisterio de Educación Infantil de la Universidad de Alicante. El objetivo del estudio era conocer las competencias digitales en cuanto al uso y dominio de las TIC por parte de futuros docentes. Como herramienta de recogida de datos se ha empleado el cuestionario validado por Guzmán (2008) Utilización de las Tecnologías de la Información y la Comunicación [TIC] en estudiantes universitarios. Los resultados obtenidos muestran un buen nivel de uso y dominio de las herramientas TIC, así como un buen nivel en la competencia digital.
\end{abstract}

Palabras clave: competencia digital, formación inicial, TIC.

\begin{abstract}
Resum
En el present treball desenvolupem l'estudi descritptiu realitzat sobre les competències digitals en alumnes de Magisteri d'Educació Infantil de la Universitat d'Alacant. L'objectiu de l'estudi ha estat conèixer les competències digitals pel que fa a l'ús i domini de les TIC per part de futurs docents. Com a eina de recollida de dades, hem fet servir el qüestionari validat per Guzmán (2008) Utilización de las Tecnologías de la Información y la Comunicación [TIC] en estudiantes universitarios. Els resultats obtinguts mostren un bon nivell d'ús i un bon domini de les eines de les TIC i, alhora, un bon domini en la competència digital.
\end{abstract}

Paraules clau: competència digital, formació inicial, TIC.

\section{Abstract}

This paper presents the results of a descriptive study on digital competences. A survey was conducted among Pre-School Education Teaching degree students. The aim of the study was to know the degree of digital competences regarding the ICT usage and skills of future teachers. As a data gathering tool we used the survey validated by Guzmán (2008) Utilización de las Tecnologías de la Información y la Comunicación $[T I C]$ en estudiantes universitarios. The results show a good level of usage and control of ICT tools and a good level of digital competence.

Keywords: digital competence, initial training, ICT.

\footnotetext{
${ }^{1}$ El presente trabajo se enmarca en el seno del Grupo de Investigación “EDUTIC-ADEl” (Ref.: Vigrob-039), del Grupo de Investigación e Innovación en Tecnología Educativa (GITE) "EDUTIC-ADEI-EDAFIS" (Ref. GITE-09004-UA), y del Programa de Redes de investigación en docencia universitaria del ICE, todos ellos de la Universidad de Alicante (UA); del proyecto Instituto Superior de Investigación Cooperativa IVITRA (Ref.: ISIC/2012/022; http://www.ivitra.ua.es) y del Proyecto DIGICOTRACAM (“Programa Prometeo de la Generalitat Valenciana para Grupos de Investigación en I+D de Excelencia”, Ref.: Prometeo2009-042, cofinanciado por el FEDER de la UE) y MICINN FFI2009-13065 y FFI2010-09064
} 


\section{Introducción}

Sin lugar a dudas, las transformaciones de la sociedad afectan de forma directa a las aulas y al papel que deben desempeñar los docentes ante la incorporación de las nuevas tecnologías en su práctica diaria, sea cual sea el contexto geográfico donde desarrolle su desempeño profesional (du Plessis y Webb 2012). En la actualidad no se puede considerar al docente sólo como un mero conocedor de su materia. Debe saber cómo gestionar la información que de ella dispone y, además, administrar dicha información y servir como orientador a sus alumnos. Una buena ayuda para realizar esta tarea es que el docente se sirva de las nuevas tecnologías. Desde este enfoque, se prevé un cambio en el rol del profesorado. El docente pasa de ser un expositor a ser un guía del conocimiento y a ofrecer los medios por los cuales acceder a la información. También se convierte en una figura guía en la gestión y utilización de la información para su correcto uso por parte de los alumnos (Gisbert 2002; Ramírez et al. 2012).

El profesor debe ofrecer la posibilidad a sus alumnos de que aprendan por ellos mismos y ofrecerse como facilitador de ocasiones para aprender; es decir, el docente debe ser capaz de preparar oportunidades de aprendizaje para sus alumnos y ellos, a su vez, capaces de ser autónomos en sus aprendizajes. En la sociedad de la información, no sólo los docentes deben adaptarse a sus alumnos y a los nuevos métodos y estrategias de enseñanza, sino también hacerlo con la manera de trabajar, tanto individual como en grupo, y con su relación con el entorno del centro, el equipo directivo y las familias (Fernández 2003).

Es evidente que el avance de la tecnología es rápido y permanente, y por ello hay que tener presente qué formación se les va a facilitar a los futuros docentes. Se debe considerar que ejercerán sus funciones en la sociedad del siglo XXI bajo una conformación entre los avances de la sociedad de la información y el conocimiento, y las necesidades propias de los alumnos para su incorporación a los diferentes ámbitos de su vida (Balanskat et al. 2006). De esta forma hay que tener presente cómo se va a formar a los docentes en relación a las TIC.

No se trata de formar a los docentes únicamente en TIC sino, más bien, formar al profesorado en la utilización de estas tecnologías para su práctica docente, y tener presentes cuestiones tales como: capacitar al docente para aplicar las estrategias adecuadas; saber cómo integrar de forma adecuada las TIC y ser realista en su aplicación; y, por último, pensar en el docente como un investigador más y no un mero consumidor de los resultados obtenidos por otros (Llorente 2008; Bates 2001).

Es en las universidades donde debe empezar este cambio, puesto que en sus aulas es donde se están formando los nuevos profesores que en el futuro desarrollaran sus funciones como docentes. En este sentido, hay que tener presente dos cuestiones importantes para fomentar de forma adecuada la formación del profesorado en TIC: por un lado, las actitudes que tienen los docentes frente a las TIC y, por otro lado, las competencias necesarias que los docentes deben tener sobre las TIC (Gisbert 2002; Prendes et al. 2010).

En 2008 la UNESCO elaboró un informe de plan de estudios con las normas sobre competencias en TIC para los docentes. Este plan está basado en tres componentes principales: nociones básicas de tecnología, profundización de conocimientos y creación de conocimientos. A su vez éstos postulan una serie de objetivos y unas competencias nece- sarias a alcanzar por los docentes. Además, son varios los autores que establecen las competencias TIC que son necesarias en los docentes (Tejedor et al. 2006; Marqués 2008b; Guzmán et al. 2008; Martínez 2009).

En este sentido, hemos tomado como referente un estudio realizado por Guzmán y Gisbert (2008) sobre las competencias digitales para llevar a cabo una investigación cuyo objetivo principal ha sido conocer el uso y dominio de las TIC en estudiantes de Magisterio de la Universidad de Alicante. En definitiva, conocer las competencias digitales de futuros docentes.

\section{Método}

\subsection{Participantes}

Los participantes de nuestro estudio son estudiantes de segundo de Magisterio de Educación Infantil de la Universidad de Alicante, que se seleccionaron tras un muestreo no probabilístico de tipo intencional (Alaminos 2006). La muestra está compuesta por un total de 61 alumnas, con edades comprendidas entre los 19 y los 44 años, siendo la media de 22,26 años (dt.: 4,412). La muestra es representativa en cuanto al subgrupo sometido a análisis, ya que en su mayoría la especialidad de Educación Infantil está compuesta por mujeres.

\subsection{Instrumento}

Para la recogida de datos se empleó el cuestionario sobre competencias digitales en universitarios elaborado por Guzmán (2008) Utilización de las tecnologías de la información y comunicación en el aprendizaje universitario. El cuestionario está dividido en cuatro bloques: (1) Datos generales del alumno; (2) Uso del ordenador; (3) Frecuencia de uso, utilidad y dominio de los medios TIC; y (4) Competencia básica en TIC. En el primer bloque se trata de conocer la edad, el género y la especialidad de Magisterio de los participantes. En el segundo bloque se les plantean cuestiones referidas al tipo de ordenador, el número de horas que lo utilizan al día, el uso que le dan y el dominio que creen que tienen del mismo de manera general. En el tercer bloque las preguntas tratan de conocer la frecuencia de uso, la utilidad del medio TIC y el dominio que creen que poseen de diferentes medios, tales como: consultar y enviar correos electrónicos, navegar por Internet, lenguaje de programación o edición de páginas Web, entre otros. Por último, el cuarto bloque está compuesto por cuatro ámbitos en relación a las competencias que se quieren estudiar. A continuación pasamos a detallar los ítems que componen cada uno de ellos:

- Alfabetización tecnológica.

- Logra autonomía delante de los problemas técnicos cotidianos (CProblem)

- Muestra interés para actualizar los conocimientos sobre los medios TIC (CConocim)

- Aprovecha los nuevos entornos virtuales de aprendizaje (CEntorno).

- Instrumentos de trabajo intelectual.

- Emplea los medios (TIC) para buscar, localizar, evaluar y recuperar información (Cbuscar).

- Aprende a trabajar en equipo en entornos de trabajo colaborativo (CColabor)

- Baja archivos (CFichero).

- Analiza y comenta críticamente páginas Web (CWeb).

- Crea un documento incorporando información textual y gráfica (CCrear). 
- Usa buscadores específicos (CBuscad).

- Evalúa de forma crítica diferentes recursos educativos (CEvaluar).

- Trabaja de manera individual (CTrabind).

- Trabaja de manera colaborativa (CTrabcol).

- Tratamiento y difusión de la información.

- Evalúa de manera crítica y responsable la información recogida (CCrítica)

- Contrasta la validez y actualidad de la información localizada (CValidez).

- Respetas las fuentes de autoría de la información buscada y tratada (CAutoría).

- Aprovecha nuevas fuentes de información y recursos para el aprendizaje (CFuentes).

- Presenta y difunde con la ayuda de los Medios TIC trabajos y conclusiones obtenidas (CDifund).

- Herramienta de comunicación.

- Participa en actividades en grupo, por ejemplo: fórum y entornos colaborativos (CFórum).

- Es consciente del estilo comunicativo y actuaciones adecuadas en un fórum (CEstilComu).

- Comprende y utiliza un entorno de trabajo colaborativo (CEntorTrb).

- Utiliza los medios TIC para trabajar, procesar la información y comunicarte (CTics).

- Interactúa con el profesor y atiende sus indicaciones (CProfe).

En los bloques 2, 3 y 4 las respuestas se puntúan en una escala Likert de 6 puntos, siendo 1 la mínima puntuación ("nunca/nada") y 6 la máxima ("siempre/mucho"). Como información adicional se añadieron dos ítems al cuestionario original sobre el tipo de ordenador y las horas al día de utilización del mismo para obtener información relativa a la frecuencia de uso del ordenador. Como coeficiente de fiabilidad para detallar la consistencia interna de los ítems se ha empleado el Alpha de Cronbach, siendo de $\alpha=.92$ en el cuestionario original y de $\alpha=.95$ en el presente estudio, lo que viene a mostrar un nivel de confianza excelente (Colás et al. 1998).

\subsection{Procedimiento}

Se elaboró el cuestionario autoadministrable en soporte digital a través de la plataforma de software libre LimeSurvey versión 1.85 . Esta plataforma permite realizar cuestionarios online en la página www.limesurvey.org, donde se albergan y se trabaja con ellos, pudiendo realizar múltiples opciones con los cuestionarios, tanto en el tipo de preguntas como en la forma de administrarlos. Una vez realizado el cuestionario, se ofrece la opción de ser enviado a los participantes del estudio y contestado online o en papel (Martín 2007). En nuestro caso, los alumnos respondieron al cuestionario online y por ello fue necesario que los alumnos pudieran disponer de ordenador y conexión a Internet. Así pues, se contactó con la profesora de Nuevas Tecnologías para que en una sesión los alumnos contestaran al cuestionario. Para ello, el cuestionario se indexó en una dirección electrónica de la web del grupo de Investigación EDUTIC-ADEI de la Universidad de Alicante.

A los participantes se les explicó el fin del estudio tanto de forma verbal como por escrito en el mismo cuestionario, y en todo momento se aseguró la confidencialidad de las respuestas.

\subsection{Diseño y análisis de datos}

Para el estudio de las competencias de los alumnos de Magisterio se ha desarrollado una investigación cuantitativa de tipo no experimental siguiendo un diseño descriptivo, ya que nuestro objetivo principal es conocer el uso y dominio de las TIC y las competencias digitales en dichos alumnos (Castejón 1997). Una vez recogidos los datos, se analizan y se presentan para describir la realidad de los participantes en cuestiones sobre la competencia digital. Todos los análisis estadísticos se han llevado a cabo con el paquete estadístico SPSS versión 17.0 (Gardner 2003).

\section{Resultados}

Después de los análisis estadísticos realizados, se obtienen los resultados que a continuación presentamos. El cuestionario consta de cuatro bloques: (1) Datos generales del alumno; (2) Uso del ordenador; (3) Frecuencia de uso, utilidad y dominio de los medios TIC; y (4) Competencia básica en TIC. A continuación presentamos los resultados obtenidos.

\subsection{Datos generales del alumno}

En este bloque se presenta la edad, el género y la carrera en curso del alumnado. El género femenino representa el $100 \%$ de la muestra, así como la especialidad de Magisterio de Educación Infantil que también representa el 100\% de la muestra estudiada. En cuanto a la edad, en la Figura 1 se presentan los valores estadísticos de esta variable.

\begin{tabular}{|c|c|c|c|c|c|}
\hline \multicolumn{5}{|c|}{ Estadísticos edad del alumnado } \\
\hline $\mathrm{N}$ & $\mathrm{M}$ & $\mathrm{Mo}$ & $\mathrm{DT}$ & $\begin{array}{c}\text { Edad } \\
\text { mínima }\end{array}$ & $\begin{array}{c}\text { Edad } \\
\text { máxima }\end{array}$ \\
\hline 61 & 22,26 & 19 & 4,41 & 19 & 44 \\
\hline
\end{tabular}

Figura 1. Valores sobre la edad de la muestra

\subsection{Uso del ordenador}

Para este bloque los datos se recogieron de las preguntas 2.1., 2.2., 2.3. y 3.1. Las preguntas 2.1. y 2.2. hacen referencia al tipo de ordenador y al número de horas diarias que se utiliza. Así, de la muestra total de alumnas $n=61$, el $24,59 \%$ $(n=15)$ de alumnas tienen ordenador de sobremesa, el $32,78 \%(n=20)$ tienen ordenador portátil, y el $42,63 \%$ $(n=26)$ respondieron que tienen tanto ordenador de sobremesa como portátil. Con estas tres opciones se completa el total de la muestra, ya que ninguna marcó tener Tablet PC ni la opción de no tener ordenador.

En cuanto a las horas dedicadas al día a utilizar el ordenador (pregunta 2.2.); en la Figura 2 se muestran los intervalos de horas frente al ordenador, así como la fre-

Horas dedicadas al uso del ordenador

\begin{tabular}{ccc}
\hline Intervalos de horas & $\mathrm{N}$ & $\%$ \\
\hline $1-2 \mathrm{~h}$. & 17 & 27,9 \\
$3-4 \mathrm{~h}$. & 32 & 52,5 \\
$5-6 \mathrm{~h}$. & 12 & 19,7 \\
\hline Total & 61 & 100,0
\end{tabular}

Figura 2. Valores sobre la frecuencia del uso del ordenador 
cuencia y porcentaje. (Ver Figura 2)

En esta pregunta también figuraban las opciones que reflejaban un uso del ordenador menos de una hora al día y de más de siete horas, pero ninguna de las dos opciones fue marcada.

Las preguntas realizadas para evaluar el uso del ordenador corresponden a la pregunta 2.3. y la pregunta 3.1. sobre el dominio que presenta el alumnado ante el ordenador. Así, en las Figuras 3 y 4 presentamos las medias de respuesta de ambas preguntas. Recordamos que la escala es de tipo Likert, siendo 1 la mínima puntuación ("nunca/nada") y 6 la máxima ("siempre/mucho").

En la Figura 3 se puede apreciar que la mayoría de los alumnos utilizan el ordenador para fines académicos (UsAcademi), con una $\mathrm{M}=5,38$; DT $=0,711$ seguido de fines como herramientas de comunicación (UsComun) con una $\mathrm{M}=4,85$; DT=1,093. En la Figura número 4, la media de respuestas está equiparada en cuanto a un dominio general (DomGeneral) y un dominio para el trabajo (DomTrabajo), y el alumnado considera tener un mejor dominio cuando lo usa como herramienta de comunicación (DomComunic) con una $\mathrm{M}=5,23$; $\mathrm{DT}=0,804$.

\subsection{Frecuencia de uso, utilidad y dominio de los} medios TIC

En la pregunta 2.4. se consulta la frecuencia de uso, la utilidad y el dominio del alumnado sobre una lista de medios TIC. Recordamos que la escala es de tipo Likert, siendo 1 la mínima puntuación ("nunca/nada") y 6 la máxima ("siempre/mucho"). Para ilustrar el modo de contestación de esta pregunta se les planteó a las alumnas el siguiente ejemplo: "Si te preguntan por el transporte que utilizas para ir a la universidad y tu caso es el siguiente: no acostumbras a ir en coche particular, pero crees que es un medio de transporte muy útil, y hace poco que aprendiste a conducir, tu valoración podría ser: frecuencia 2 , utilidad 6, y dominio 3." En la Figura número 5, se presentan los resultados de cada consulta así como el total de cada ítem (ver Figura 5)

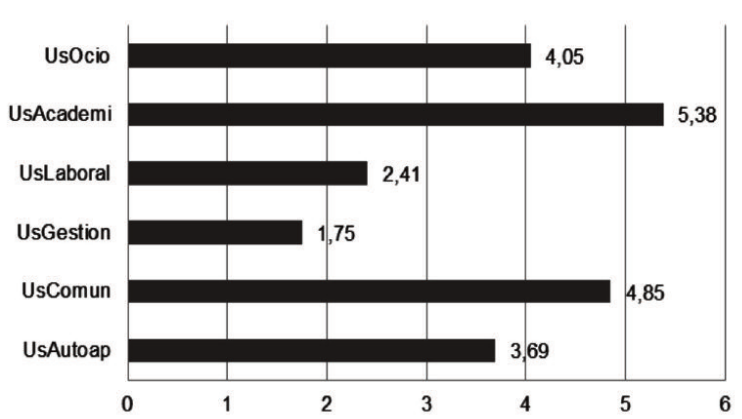

Figura 3. Medias de respuesta sobre uso del ordenador.

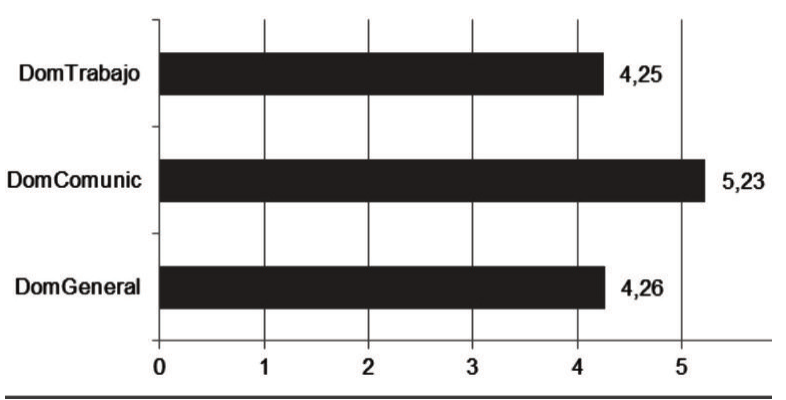

Figura 4. Medias de respuesta para el dominio del ordenador
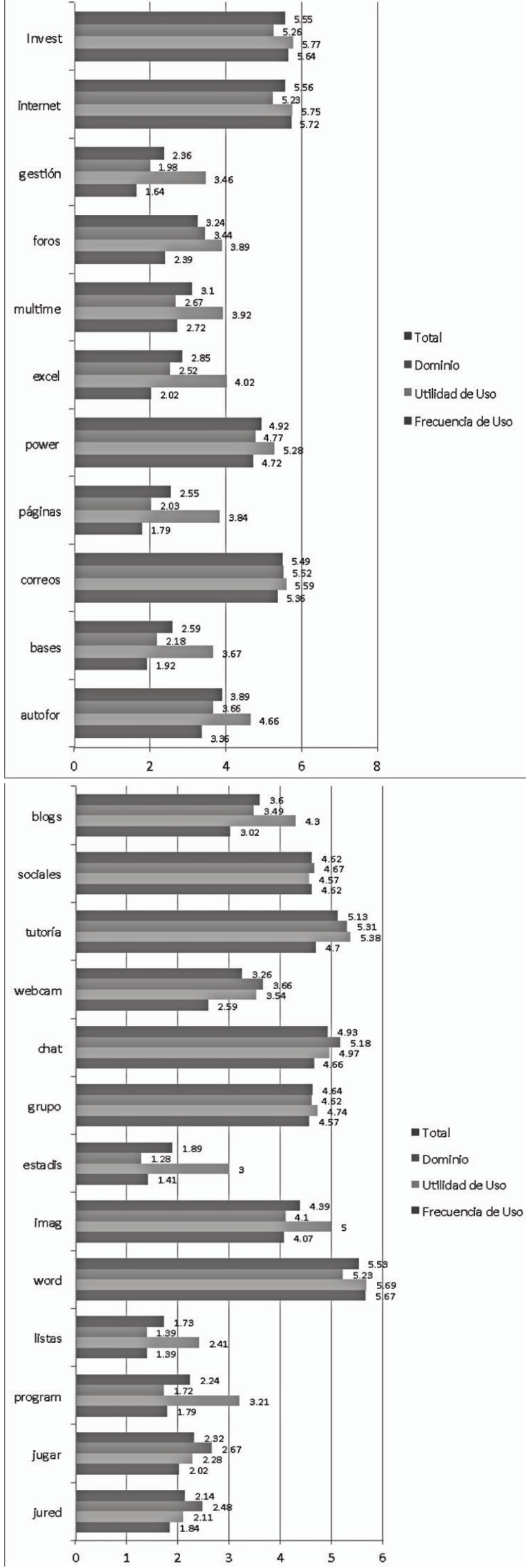

Figura 5. Medias de respuesta para la frecuencia, uso y dominio de medios TIC 
Observando los totales de los ítems, vemos que los medios que más manejan son los relacionados con Tutoría profesoralumno (tutoría) $\mathrm{M}=5,13$; DT=0,994; Consultar y enviar correos electrónicos (correos) $\mathrm{M}=5,49 ; \mathrm{DT}=0,751 ;$ Procesador de textos (word) $\mathrm{M}=5,53$; DT=0,875; Investigación y búsqueda de información (invest) $\mathrm{M}=5,55 ; \mathrm{DT}=0,79 ; \mathrm{y}$ Navegar por Internet (internet) $\mathrm{M}=5,56 ; \mathrm{DT}=0,71$. Por el contrario, los medios que menos emplean son los relacionados con Listas de distribución (listas) M=1,73; DT=1,03 y Programas estadísticos (estadis) $\mathrm{M}=1,89$; DT $=1,24$.

\subsection{Competencia básica en TIC}

En este bloque tratamos de conocer las competencias digitales que las alumnas de Magisterio de Educación Infantil tienen. Para ello, se clasificaron de acuerdo a cuatro ámbitos: a. Alfabetización tecnológica; b. Instrumento de trabajo intelectual; c. Tratamiento y difusión de la información; y d. Herramientas de comunicación.

En la Figura número 6, podemos ver las medias de respuesta del alumnado sobre competencias digitales en el ámbito de alfabetización tecnológica. A continuación se listan las competencias consideradas. Recordamos que la escala es de tipo Likert, siendo 1 la mínima puntuación ("nunca/nada") y 6 la máxima ("siempre/mucho") (Ver Figura 6).

Se puede apreciar que la competencia mejor valorada por el alumnado es cuando Muestra interés para actualizar los conocimientos sobre los medios TIC (Cconocim), $\mathrm{M}=4,59 ; \mathrm{DT}=1,086$.

En la Figura 7 se refleja la media de respuestas sobre cómo se valora el alumnado en relación a las competencias digitales en el ámbito de instrumentos de trabajo intelectual (Ver Figura 7).

En esta figura se aprecia que el alumnado considera tener un mayor nivel de competencia cuando trabaja indi-

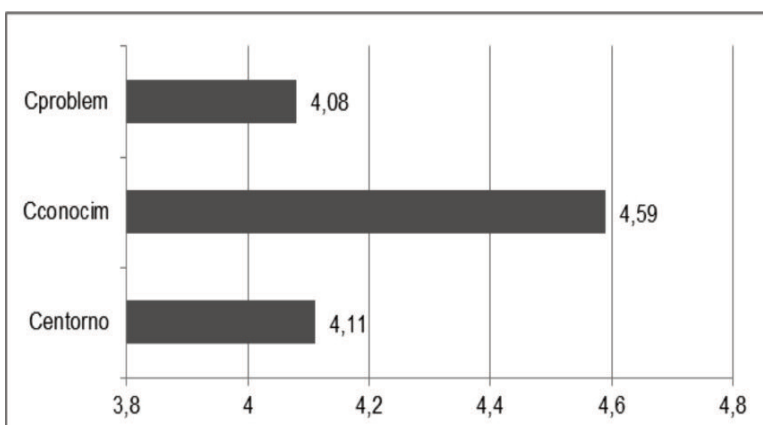

Figura 6. Medias de respuesta para la competencia digital en alfabetización tecnológica

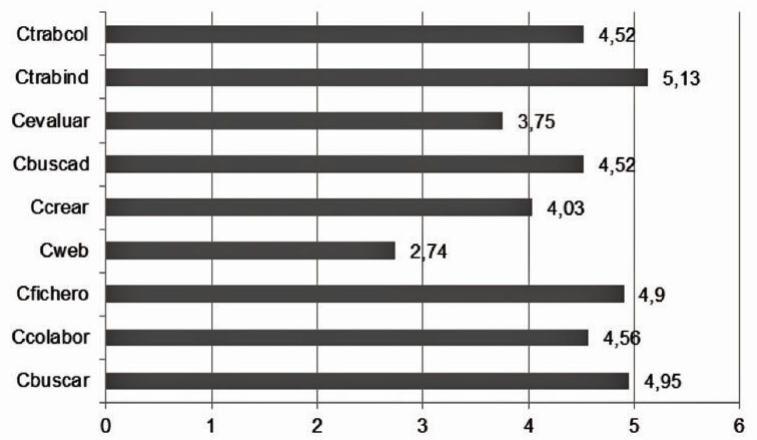

Figura 7. Medias de respuesta en la competencia digital en instrumento de trabajo intelectual vidualmente (Ctrabind) $\mathrm{M}=5,13 ; \mathrm{DT}=0,741$ seguido de buscar, localizar, evaluar y recuperar información (Cbuscar) y bajas archivos (CFichero), con unos resultados de $\mathrm{M}=4,95$; DT= 0,973 y M=4,9; DT=1,136 respectivamente. En contra, la competencia menos valorada es la de analizas y comentas críticamente páginas Web (CWeb), M=2,74; DT=1,25.

En la Figura número 8 se muestran las medias de las respuestas sobre las competencias en el ámbito de tratamiento y difusión de la información (Ver Figura 8).

La competencia que mejor ha sido valorada es la referente a aprovechas nuevas fuentes de información y recursos para el aprendizaje (CFuentes) $\mathrm{M}=4,92 ; \mathrm{DT}=0,918$. Las otras competencias tienen una valoración similar entre ellas.

En la siguiente figura se muestran las medias de las respuestas obtenidas en la competencia digital correspondiente a herramientas de comunicación (Ver Figura 9).

En la Figura 9 podemos observar que la competencia mejor valorada por los estudiantes es la referente a Utilizas los Medios TIC para trabajar, procesar la información y comunicarte (CTics), M=5,12; DT =0,915 seguida de Interactúas con el profesor y atiendes sus indicaciones (CProfe), $\mathrm{M}=4,72 ; \mathrm{DT}=1,127$. Las otras tres competencias están próximas en cuanto a la valoración hecha.

Por último, presentamos las tres competencias que han sido mejor valoradas por los alumnos: en primer lugar, la competencia en relación a trabajas de manera individual (CTrabind), $\mathrm{M}=5,13$; DT=0,741; en segundo lugar, utilizas los medios TIC para trabajar, procesar la información y comunicarse (CTics), $\mathrm{M}=5,12 ; \mathrm{DT}=0,915 ; \mathrm{y}$, en último lugar, la competencia empleas los medios TIC para buscar, localizar, evaluar y recuperar información (CBuscar) con una M=4,95; DT=0,973.

\section{Discusión y conclusiones}

Todas estas respuestas nos llevan a concluir que los alumnos encuestados se valoran de forma satisfactoria en

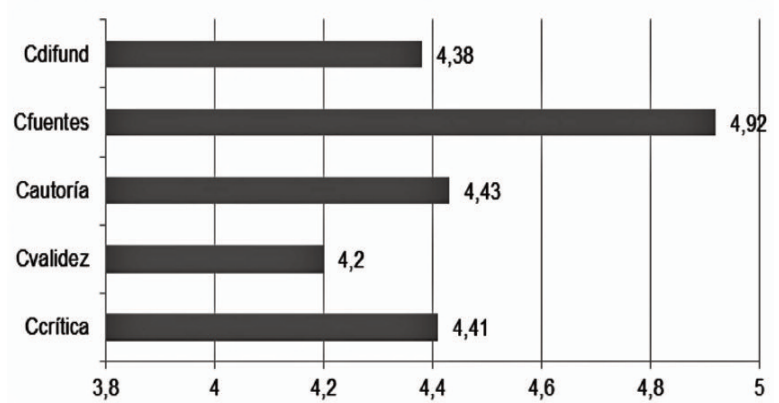

Figura 8. Medias de respuesta de la competencia digital en tratamiento y difusión de la información

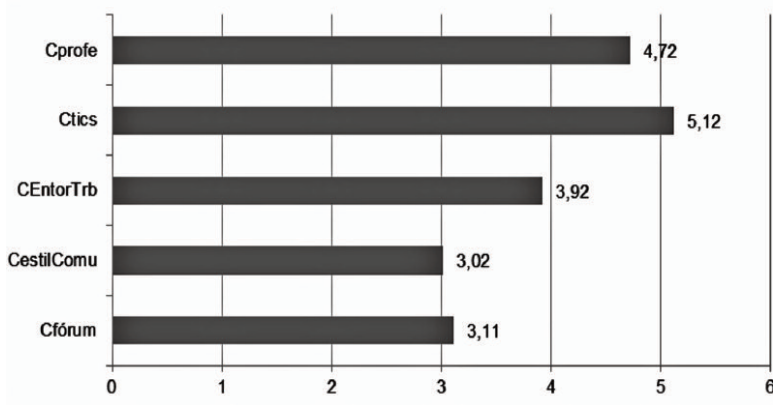

Figura 9. Medias de respuesta de la competencia digital en herramienta de comunicación 
aquellas actividades que tienen que ver con el empleo de los medios TIC a la hora de comunicarse, tanto en su uso y dominio, como en el nivel de competencia, así como en actividades de aprendizaje y autoaprendizaje en entornos virtuales y en tareas de manejo de información, todo ello haciendo uso de Internet. Se observa así que cuentan con la formación necesaria para realizar todo este tipo de actividades.

Son varios los estudios llevados a cabo relacionados con esta materia (Prendes et al. 2010; Solesa y Solesa-Grijak 2011; Suarez-Rodriguez et al. 2012). Los resultados hallados en nuestro estudio se relacionan con un estudio llevado a cabo por Guzmán y Gisbert (2008) en el que también se plantearon conocer los usos digitales y el nivel de competencia digital no sólo de alumnos de Magisterio sino de 326 estudiantes de varias titulaciones de la Universidad Autónoma de Querétaro, México. En su estudio, el uso y las competencias digitales de los alumnos también obtuvieron un alentador promedio de respuestas positivas. En otro estudio realizado por Henríquez y Organista (2009) en el que pretendían conocer los tipos y niveles de uso tecnológico de estudiantes de nuevo ingreso en la universidad, se concluye que los resultados en cuanto al nivel de uso de la tecnología es elevado, ya que el $74 \%$ de 438 estudiantes encuestados se sitúa en el grado de mayor dominio. En otros estudios llevados a cabo en relación a las competencias digitales de los estudiantes universitarios también concluyen con resultados similares a los obtenidos en nuestro estudio (Guitert et al. 2008; Lerma et al. 2008). En vista de estos resultados podemos afirmar que un gran porcentaje de los estudiantes universitarios tienen buen nivel en cuanto a la competencia digital. En el caso de los estudiantes de magisterio estudiados en esta investigación, dicha competencia adquiere un significado añadido y debe ser conocida y utilizada de forma óptima, ya que posteriormente serán ellos los transmisores de conocimiento a sus futuros alumnos.

Consideramos que hemos conseguido los objetivos que nos planteábamos con la realización de la investigación en el grupo estudiado: conocer el uso de las TIC en los estudiantes de magisterio seleccionados y saber las competencias en TIC que dichos estudiantes tienen como futuros docentes. De ningún modo pretendemos generalizar dichos resultados sino simplemente apuntar los resultados obtenidos en la investigación que hemos realizado. En términos generales los resultados han sido satisfactorios y están en consonancia con los que esperábamos obtener. En primer lugar, la edad de los participantes se encuentra entre los 19 y los 44 años. Si bien el 29,5\% (n=18) de la muestra tiene 19 años y un 19,7\% (n=12) tiene, es importante considerar que la edad no ha da suponer un obstáculo para seguir con la formación académica en estudios universitarios. Sí es cierto, en cambio, que a mayor edad de los encuestados el porcentaje de participantes se va reduciendo. Así pues el 9,8\% $(n=6)$ son alumnos con 22 y 23 años, el 4,9\% $(n=3)$ alumnos con 28 años y, la persona con más edad, de 44 años, representa $1,6 \%(n=1)$ de la muestra.

En el segundo bloque Uso del ordenador, las preguntas 2.1. y 2.2. trataban de dar respuesta al tipo de ordenador y horas dedicadas a su uso al día. Así encontramos un porcentaje elevado de alumnos que de manera personal tienen ordenador de sobremesa (OrPC) y también portátil (OrPortátil) con un 42,63\% ( $\mathrm{n}=26)$ y en cuanto a las horas dedicadas a su uso al día, el intervalo de horas con mayor porcentaje ha sido el de 3 -4horas, con un $52,5 \%(n=32)$. Las horas empleadas en el uso del ordenador están comprendi- das entre 1 y 6 horas, ya que no hubo respuestas seleccionadas que reflejen un uso de menos de 1 hora ni de más de 7 . Se pone de manifiesto, por tanto, el uso frecuente del ordenador por parte de los estudiantes, tanto para responder a cuestiones académicas como personales. Por otro lado, queda comprobado que tienen a su disposición un ordenador de mesa y/o portátil para poder desarrollar sus actividades académicas o de otra índole. Debemos tener en cuenta este hecho y considerar que los estudiantes cuentan con los medios necesarios para estar al corriente de todo lo relacionado con su formación, ya que muchos materiales y recursos para el aprendizaje de las asignaturas están disponibles vía online a través del Campus Virtual, como también la comunicación mediante tutoría y/o correo electrónico entre profesores y alumnos. En las otras dos preguntas que componen este bloque, preguntas 2.3. y 3.1., se les preguntaba sobre el uso y dominio del ordenador, en las que las variables mejor valoradas han sido las referentes a cuestiones académicas (UsAcademi) y de comunicación (UsComun y DomComunic). Se resalta así nuevamente la importancia de disponer de los recursos necesarios para establecer una relación online con la enseñanza y el aprendizaje de las asignaturas; es decir, se pone de manifiesto la valoración positiva que tienen los estudiantes acerca de establecer nuevas maneras de comunicación y de estar al día sobre las facilidades que las TIC pueden ofrecer a su futuro laboral.

En el tercer bloque, Frecuencia de uso, utilidad y dominio de los medios TIC, pregunta 2.4., compuesto por un total de 24 ítems, el $62 \%(n=15)$ se valoraron con una puntuación de 3 ó superior (recordamos que la escala era de 1 a 6). Se puede decir, por tanto, que los alumnos han valorado de forma satisfactoria su propia formación en cuanto a medios TIC. Los dos ítems que mejor puntuación han obtenido han sido el de Navegar por Internet (internet) e Investigación y búsqueda de información (invest), dos tareas que no sería posible llevar a cabo sin una de las facilidades más significativas del colectivo de las herramientas TIC como es la conexión a Internet (Marqués 2008a), que es esencial que los alumnos dominen y que dispongan de una correcta formación sobre su uso. Por el contrario, las preguntas que menos ha valorado el alumnado han sido las referentes a aquellos medios TIC en los que su uso no es tan frecuente, como por ejemplo en programas estadísticos (estadis) y en listas de distribución (listas). De hecho gran parte del alumnado desconocía el funcionamiento de las listas de distribución, y quizá esto nos hace plantearnos la posibilidad de incluir una opción de no sé que es a la hora de elegir una respuesta. Esta cuestión se tendrá presente para la realización de futuras investigaciones relacionadas con el tema de estudio, pero en este caso y como en páginas anteriores se menciona, el cuestionario fue cedido del estudio realizado por Guzmán (2008).

En el último de los bloques, el número 4 sobre las competencias digitales, del total de los ítems planteados $(\mathrm{n}=22)$ el 77,27\% ( $n=17)$ ha sido valorado por los alumnos con una puntuación de 4 o superior (siendo la escala de 1 a 6), lo que viene a mostrar que consideran tener un nivel competente para el desarrollo de su trabajo como docentes. Como anteriormente se ha expuesto, las tres competencias cuya valoración ha sido mayor han sido las siguientes: trabajas de manera individual (CTrabind), en segundo lugar utilizas los medios TIC para trabajar, procesar la información y comunicarse (CTics) y, en último lugar, la competencia empleas los medios TIC para buscar, localizar, evaluar y recuperar 
información (CBuscar). Sorprende que la competencia mejor valorada haya sido la relacionada con el trabajo individual, ya que muchos de los trabajos que se realizan en la carrera son en grupo para fomentar de esta forma el trabajo colaborativo entre los estudiantes. Por el contrario, las tres competencias que han obtenido una valoración inferior a 4 han sido las siguientes: analizas y comentas críticamente páginas Web (CWeb), participas en actividades en grupo, por ejemplo: fórum y entornos colaborativos (Cfórum) y eres consciente del estilo comunicativo y actuaciones adecuadas en el fórum (CEstilComu). El motivo de que estas competencias hayan sido peor valoradas se puede deber a que los alumnos piensen que no están tan directamente relacionadas con el desarrollo de las asignaturas y con su posterior trabajo. No obstante, en el desarrollo de la labor docente es muy importante participar en entornos de colaboración y compartir experiencias, por lo que resulta conveniente saber cómo comunicarse dentro de estos entornos.

No quisiéramos finalizar sin indicar las limitaciones que hemos encontrado en el estudio. La primera hace referencia a las características de la muestra en cuanto al género, ya que todos los participantes han sido mujeres. Otra de las limitaciones ha sido en relación a la especialidad de Magisterio, ya que toda la población pertenecía a la misma especialidad, impidiendo de esta forma generalizar los resultados al resto de especialidades del mismo curso. Por tanto, como futuras líneas de investigación, nos proponemos realizar el estudio de forma más amplia, incluyendo al resto de especialidades de Magisterio y así aumentando la muestra objeto de estudio y poder establecer comparaciones entre unas especialidades y otras e incluyendo de esta forma ambos géneros para su posible comparación.

Por último, queremos añadir unas palabras de algunas de las alumnas encuestadas para este estudio que ponen de manifiesto sus impresiones sobre la materia y la importancia que tiene ser competente en cuanto a cuestiones tecnológicas para en un futuro estar preparadas para su tarea docente: "En mi opinión, es muy importante que un maestro o maestra conozca y utilice las TIC, porque los alumnos y sus necesidades son diferentes de las que había hace diez años. Es fundamental que la información que se presenta en el aula esté relacionada con lo que pase en nuestro mundo real, la tecnología forma parte de nuestras vidas". Otra alumna aportaba la siguiente reflexión: "Creo que en la sociedad de la información en la que vivimos actualmente es necesario que un maestro conozca y sepa utilizar correctamente y de manera adecuada las TIC. Utilizando las nuevas tecnologías podemos incentivar la motivación de los alumnos. Y si las utilizamos correctamente, nos brindan muchos recursos que podemos utilizar en el aula".

Consideramos, por tanto, que es esencial que los alumnos sean conscientes de su necesidad, como futuros docentes, de adquirir la formación adecuada en materia de competencias digitales para ofrecer una educación de calidad a sus alumnos contextualizada en la sociedad actual: la sociedad de la información.

\section{Bibliografía}

Alaminos, Antonio (2006). El muestreo en la investigación social. En A. Alaminos y J.L. Castejón, Elaboración, análisis e interpretación de encuestas, cuestionarios y escalas de opinión. Alicante: Marfil. Pp. 41-55

Balanskat, A., Blamire, R. \& Kefala, S. (2006). The ICT Impact Report. A review of studies of ICT impact on scho- ols in Europe. European Schoolnet, European Comission. http://ec.europa.eu/education/doc/reports/doc/ictimpact.pdf Fecha de consulta, 10.09.2012

Bates, Tony (2001). Cómo gestionar el cambio tecnológico. Barcelona: Gedisa.

Castejón, Jose Luís (1997). Introducción a los métodos y técnicas de investigación y obtención de datos en psicología. Alicante: Ediciones Club Universitario.

Colás, Pilar; Buendía, Leonor (1998). Investigación educativa. Sevilla: Alfar.

Du Plessis, André; Webb, Paul (2012). A teacher proposed heuristic for ict professional teacher development and implementation in the south african context. Turkish online journal of educational technology, 11(4), 46-55. http://www.tojet.net/articles/v11i4/1144.pdf

Fernández, Ricardo (2003). Competencias profesionales del docente en la sociedad del siglo XXI. Organización y gestión educativa: Revista del Fórum Europeo de Administradores de la Educación, 1 (11), http://dialnet.unirioja.es/servlet/articulo?codigo=29259o Fecha de consulta, 16.08.2012

Gardner, Robert. (2003). Estadística para Psicología usando SPSS. Madrid: Pearson

Gisbert, Mercé (2002). El nuevo rol del profesor en entornos tecnológicos. Revista Acción Pedagógica, 11(1), http://www.saber.ula.ve/handle/123456789/17053 Fecha de consulta, 21.09.2012

González, Angel (2000). Nuevas tecnologías y formación contínua. Algunos elementos para la reflexión. En J. Cabero et al. (Coord.) Y continuamos Avanzando. Las Nuevas Tecnologías para la mejora educativa (pp.315330). Sevilla: Editorial Kronos.

Guitert, Montse; Guerrero, Ana; Ornellas, Adriana; Romeu, Teresa; Romero, Marc (2008). Implementación de la competencia transversal "Uso y aplicación de las TIC en el ámbito académico y profesional” en el contexto universitario de la OUC. Revista Latinoamericana de Tecnología Educativa. RELATEC, 7 , http://dialnet.unirioja.es/servlet/articulo?codigo $=2860446$ Fecha de consulta, 13.07.2012

Guzmán, Teresa (2008). Las tecnologías de la información y la comunicación en la Universidad Autónoma de Querétaro: propuesta estratégica para su integración. Tesis doctoral. Universitat Rovira i Virgili, http://www.tdx.cat/handle/10803/8937 Fecha de consulta, 28.07.2012

Guzmán, Teresa; Gisbert, Mercé (2008). Competencias TIC de los estudiantes de la Universidad Autónoma de Querétaro (UAQ): un estudio de caso, www.virtualeduca.info/zaragozao8/.../Competencias-

VirtualEo8.doc Fecha de consulta, 13.09.2012

Henríquez, Patricio; Organista, Javier (2009). Definición y estimación de tipos y niveles de uso tecnológico: una aproximación a partir de estudiantes de recién ingreso a la universidad. EDUTEC, Revista Electrónica de Tecnología Educativa, 30, 1-20,

http://edutec.rediris.es/revelec2/revelec3o/ Fecha de consulta, 04.09.2012

Lerma, Silvia; Lara, Juan Víctor Manuel; Anguiano, Víctor Manuel (2008). Competencias en tecnologías de la información y de la comunicación en estudiantes de la licenciatura de medicina del centro universitario de ciencias de la salud. Centro universitario de Ciencias de la Salud. Universidad de Guadalajara. 
http://www.virtualeduca.info/zaragozao8/ponencias/313/Competencias\%20\%20en\%20Tecnolog\%EDa s\%20\%20de\%2ola\%20Informaci\%F3n\%20y\%20de\%2 ola\%20Comunicaci\%F3n_2.doc

Llorente, María del Carmen (2008). Aspectos fundamentales de la formación del profesorado en TIC. Pixel-Bit. Revista de Medios y Educación, 31, pp.121-130. http://www.sav.us.es/pixelbit/pixelbit/articulos/n31/ n31art/art319.htm Fecha de consulta, 23.08.2012

Marqués, Pere (2008a). La cultura tecnológica en la sociedad de la información, http://www.pangea.org/peremarques/si.htm Fecha de consulta, 10.09.2012

Marqués, Pere (2008b). Las TIC y sus aportaciones a la sociedad, http://peremarques.pangea.org/tic.htm Fecha de consulta, 16.09.2012

Martín, Carlos Juan (2007). Manual de usuario, plataforma de encuestas en línea, LimeSurvey, http://eval.posgrado.una.ac.cr/uso_de_limesurvey.pd f Fecha de consulta, 14.09.2012

Martínez, Francisco José (2009). Las tecnologías de la información y la comunicación (TIC) y las competencias básicas en educación. Espiral. Cuadernos del profesorado, 2(3), 15-26, http://www.cepcuevasolula.es/espiral/articulos/ESPIRAL_VOL_2_N_3_ART_2.pdf Fecha de consulta, 24.08.2012

Prendes, $\mathrm{M}^{\mathrm{a}}$. Paz., Castañeda, Linda; Gutiérrez, Isabel
(2010). ICT Competences of Future Teachers. Comunicar, 35, pp.175-182.

http://www.revistacomunicar.com/index.php?contenido $=$ detalles $\&$ numero $=35 \&$ articulo $=35-2010-21$ (DOI: 10.3916/C35-2010-03-11).

Ramírez, Elena; Cañedo, Isabel; Clemente, María (2012). Attitudes and Beliefs of Secondary Teachers about Internet Use in their Classrooms. Comunicar, 38, pp.147155.

http://www.revistacomunicar.com/pdf/preprint/38/En -16-PRE-12643.pdf (DOI: 10.3916/C38-2012-03-06).

Suárez-Rodríguez, Jesús M.; Almerich, Gonzalo; DíazGarcía, Isabel; Fernández-Piqueras, Rodrigo (2012). ICT Competences of teachers. Influence of personal and contextual factors. Universitas psychologica, 11 (1), 293-309. http://www.doaj.org/doaj?func=abstract\&id $=1131581$

Tejedor, Francisco Javier; García-Valcárcel, Ana (2006). Competencias de los profesores para el uso de las TIC en la enseñanza: análisis de conocimientos y actitudes. Revista Española de Pedagogía, 233, 21-43.

UNESCO (2008). Estándares TIC para la formación inicial docente: una propuesta en el contexto chileno. Chile: Ediciones UNESCO, http://www.eduteka.org/modulos/11/343 Fecha de consulta, 11.07.2012 